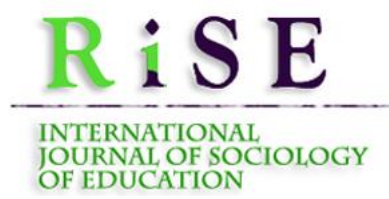

Hipatia Press

www.hipatiapress.com

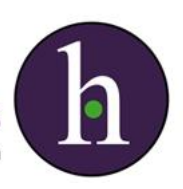

Instructions for authors, subscriptions and further details:

http://rise.hipatiapress.com

\title{
Coaching Universitario: Propuesta de un Sistema de Indicadores para su Medición
}

Jose Luis Paniza Prados ${ }^{1}$

Inmaculada Puertas Cañaveral ${ }^{1}$

Jose María Molina Morales ${ }^{2}$

1) Universidad de Granada, Spain

2) Escuela de Formación Integral en Coaching, Spain

Date of publication: February $25^{\text {th }}, 2019$

Edition period: February 2019-June 2019

To cite this article: Paniza Prados, J.L., Puertas Cañaveral, I., Molina Morales, J.M. (2019). Coaching Universitario: Propuesta de un Sistema de Indicadores para su Medición, International Journal of Sociology of Education, 8(1), 75-102. doi: 10.17583/rise.2019.3877

To link this article: http://dx.doi.org/10.17583/rise.2019.3877

\section{PLEASE SCROLL DOWN FOR ARTICLE}

The terms and conditions of use are related to the Open Journal System and to Creative Commons Attribution License (CC-BY) 


\section{University Coaching: Proposal of a Measuring Indicator \\ System}

Jose Luis Paniza Prados

Universidad de Granada

Jose María Molina Morales

Escuela de Formación Integral en Coaching

(Received: 8 November 2018; Accepted: 2 January 2018; Published: 25 February 2019)

\section{Abstract}

This article presents a teaching innovation project, funded by the University of Granada. The main objective is the efficiency measuring of a coaching process. In this way it has been designed a system of indicators that aims to be a measurement tool in two fundamental aspects: first, the acquisition of personal skills and the empowerment of self-knowledge; and second, the academic performance and the improvement of their personal potential. The structure is articulated in the following sections: first, an approximation and delimitation of the coaching concept; secondly, it analyses the application of coaching in the university; third, it explains the stages of the research design carried out; fourth, it presents the results related with the validity and reliability of the integrated indicator system; finally, the main conclusions are focus on the design of a tool that measures the effectiveness of coaching in socio-affective, socio-cognitive, executive, social, and identitive aspects with the institution and the communicative ones.

Keywords: coaching, validity and reliability, publics universities, teaching innovation. 


\section{Coaching Universitario: Propuesta de un Sistema de Indicadores para su Medición}

Jose Luis Paniza Prados

Universidad de Granada
Inmaculada Puertas Cañaveral

Universidad de Granada

Jose María Molina Morales

Escuela de Formación Integral en Coaching

(Recibido: 8 Noviembre 2018; Aceptado: 2 Enero 2019; Publicado: 25

Febrero 2019)

\section{Resumen}

Este artículo presenta un proyecto de innovación docente, financiado por la Universidad de Granada, que tiene por objetivo principal la medición de la eficacia de un proceso de coaching. Para ello se ha generado un sistema de indicadores que pretende ser una herramienta de medición en dos aspectos fundamentales: primero, la adquisición de habilidades personales y la potenciación del autoconocimiento; y segundo, el rendimiento académico y la mejora de su potencial personal. La estructura se articula en los siguientes apartados: en primer lugar, se realiza una aproximación y delimitación del concepto de coaching; en segundo término, se analiza la aplicación del coaching en el ámbito universitario; tercero, se explicita las etapas del diseño de la investigación llevado a cabo; en cuarto lugar, se presentan los resultados relativos al análisis de la validez y fiabilidad del sistema de indicadores propuesto; $y$, por último, se sintetizan las principales conclusiones centradas en el diseño de una herramienta que mide la eficacia del coaching en aspectos socioafectivos, sociocognitivos, ejecutivos, sociales, identificativos con la institución y comunicativos.

Palabras clave: coaching, validez y fiabilidad, universidades públicas, innovación docente. 


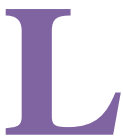

a irrupción del coaching en el mundo de las organizaciones, del desarrollo personal y del ámbito educativo en los últimos quince años, ha provocado una difusión del concepto y una normalización en el vocabulario habitual de nuestra sociedad que no queda exento de indefinición. Además, está experimentando una fase de consolidación que sugiere una clara precisión de sus habilidades y competencias, de su código deontológico y, sobre todo, de sus directrices de calidad y excelencia.

El origen del coaching como disciplina surge en Estados Unidos en el ámbito del deporte, que es la base sobre la que se sustenta el coaching actual. Las acepciones y los estilos se han ido diversificando, aunque todos confluyen, o deberían confluir, en un origen común relacionado con la filosofía y más concretamente con Sócrates. El coaching adopta de este filósofo el método de facilitar el aprendizaje a través de la toma de conciencia (para conseguir un objetivo) y del planteamiento de las preguntas correctas para que la persona pueda llegar a encontrar sus propias respuestas y, por ende, conseguir su meta. En este sentido es preciso matizar que el coaching no debe ser identificado con una terapia, consultoría o mentoring (Coutu y Kauffman, 2009), ni tampoco con un proceso curativo ni un asesoramiento psicológico. Coaching y mentoring se diferencian en que mientras el primero está directamente interesado en la mejora inmediata del desempeño y el desarrollo de habilidades mediante una forma de tutoría o instrucción, el segundo está relacionado con la adquisición de habilidades a largo plazo en el curso de una carrera en desarrollo mediante una forma de asesoramiento y orientación (Parsloe, 1992).

Para su correcta delimitación tomamos como referentes diversos autores y expertos en la materia que nos permiten precisar diferentes tendencias tanto para su definición como para su ámbito de actuación.

La primera tendencia se centra en el logro del rendimiento de la persona y se podría definir como el proceso en el que se ayuda a las personas a rendir al máximo de sus posibilidades. Esto implica hacer aflorar todo el potencial del individuo, ayudarle a superar sus barreras y limitaciones personales para alcanzar lo mejor de sí, y facilitarle el modo de funcionar más eficaz. Incluso se podría diferenciar entre un coaching con "c" minúscula para trabajar el comportamiento (toma de conciencia y desarrollo de la competencia consciente) y un coaching con " $\mathrm{C}$ " mayúscula que se concentra en reforzar la identidad y los valores (Dilts, 2004). En esta línea se explica cómo el 


\section{Lozano-Pérez \& Trinidad-Requena-Capital Cultural}

coaching puede liberar el potencial de las personas, para que puedan llevar su rendimiento al máximo (Gallwey, 2006), y en el mundo de la empresa como una poderosa herramienta para el desarrollo del liderazgo y la liberación del potencial de los empleados que permitan incrementar al máximo su desempeño (Whithmore, 2011).

La segunda línea da protagonismo a la importancia del aprendizaje, así el coaching se define como un proceso sistemático de aprendizaje, focalizado en la situación presente y orientado al cambio (Bou, 2007). Para ello se facilitan los recursos y herramientas de trabajo específicas y necesarias que posibiliten el cumplimiento de las demandas personales junto a un mayor autoconocimiento y mejora de la calidad de vida. Por tanto, este aprendizaje puede generar numerosos resultados: evolución y cambios de comportamientos sostenidos en el tiempo, con acciones y reflexiones continuas (Correa, 2008); desarrollo de altas habilidades y capacidades socioemocionales y la adquisición de competencias profesionales funcionales (Medina y Perichon, 2008; y Sánchez-Teruel, 2009); y transformación tanto del tipo de observador que somos del mundo como de lo que somos de uno mismo (Wolk, 2009).

La tercera tendencia es la más ontológica y se centra en el concepto de autoconsciencia y en la necesidad de autorrealización del individuo, imprescindibles para el logro del pleno potencial, la autoexpresión y el crecimiento de la persona (Zeus y Skiffington, 2004). Considera el coaching como una dinámica de transformación mediante la cual las personas y organizaciones revisan, desarrollan y optimizan sus formas de ser y estar en el mundo, creando a través de la conversación una nueva cultura (Echeverría, 2013). Desde esta perspectiva, se busca llevar a terrenos diferentes el tipo de resultados que generaba el coach deportivo.

Para cerrar esta aproximación conceptual atendemos a las definiciones aportadas desde los principales organismos que regulan esta actividad. En primer lugar, la International Coaching Federation (ICF), define coaching como un proceso de acompañamiento reflexivo y creativo con clientes que les inspira a maximizar su potencial personal y profesional (ICF, 2016). Y en segundo, la Asociación Española de Coaching (ASESCO), que considera el coaching como un proceso de entrenamiento personalizado y confidencial mediante un gran conjunto de herramientas que ayudan a cubrir el vacío existente entre donde una persona está ahora y donde desea estar (ASESCO, 
2016).

Por último, y a modo de síntesis, desde nuestra perspectiva de análisis entendemos que el coaching se puede considerar como la disciplina que permite el desarrollo de las potencialidades intrínsecas que cada persona o grupos de personas poseen, por medio de un acompañamiento profesional hacia las metas propuestas.

\section{Coaching en el Ámbito Universitario}

La palabra proceso contiene las ideas de acción de ir hacia delante, transcurrir del tiempo y de conjunto de fases sucesivas, todas ellas muy relacionadas con el coaching como propuesta de acompañamiento al cambio. En un proceso de coaching se establece una relación profesional entre un coach y un coachee o cliente (persona o grupo de personas que reciben coaching), y tiene como objetivo principal que el coachee consiga un objetivo o meta definido previamente.

El proceso de coaching, en general, consta de un número determinado de sesiones, por lo que la duración es finita en el tiempo y dependerá del cliente y de su ritmo en el logro del cambio deseado. Se establece un contrato formal que incluye cláusulas de confidencialidad, el objetivo del coachee, que puede abarcar temas relacionados con el comportamiento, liderazgo, visión para los negocios, mejora en el desempeño laboral, etc., y áreas tácitas como creencias personales, suposiciones arraigadas, perspectivas internas, autodescubrimiento, aprendizaje profundo y cambios en el sistema de valores interno y el patrón de comportamiento (De Meuse et al., 2009). En este sentido conviene precisar que hay habilidades instaladas en valores fundamentales que cada coach debe adquirir para el ejercicio del coaching, como la honestidad, el respeto a las ideas ajenas, la tolerancia, la paciencia, la solidaridad, la comprensión y la humildad (Ravier, 2005).

El coaching aplicado al ámbito universitario puede ser una herramienta de ayuda en la consecución de las metas y objetivos de los estudiantes, adaptándose a las novedades que ha supuesto la puesta en marcha del Plan Bolonia. La enseñanza universitaria ha tenido que incorporar numerosas medidas en la formación para poder adaptarse a las directrices marcadas. Este nuevo modelo universitario europeo apuesta por una formación competencial donde las asignaturas juegan un papel clave en la formación 


\section{Lozano-Pérez \& Trinidad-Requena-Capital Cultural}

presencial, el trabajo dirigido y el aprendizaje autónomo. El alumnado es el centro del proceso educativo y el objetivo es la consecución de una enseñanza superior de mayor calidad y una mejora metodológica de las propuestas formativas.

Es precisamente en el proceso educativo, donde el coaching puede tener importantes resultados para el alumnado universitario, ya que promueve la autonomía a través de metas personales y acciones planificadas, desarrolla el autoaprendizaje y habilidades complejas y otras competencias intra e interpersonales (Sánchez Mirón et al., 2014). Se proporciona por tanto el enfoque y la conciencia necesarias para alcanzar su meta, además de cumplir con uno de los objetivos claves en el Plan Bolonia, el uso del conocimiento adquirido hacia la resolución de situaciones, y no simplemente hacia la adquisición de datos. La trascendencia en el ámbito educativo gana importancia a la hora de trabajar competencias como la comunicación y la toma de decisiones. De ahí el interés por incorporarlo como experiencia piloto en la formación de los estudiantes universitarios y en las aulas.

Las investigaciones que inciden en el proceso de aprendizaje utilizando la metodología del coaching en el ámbito educativo son relativamente recientes, y abarcan áreas de estudio como la motivación, el rendimiento académico, el aprendizaje, la toma de decisiones, las competencias personales, la inteligencia emocional, la empatía, etc.

Los primeros estudios han mostrado que, dentro de los sistemas educativos, pueden implementarse nuevos modelos de trabajo basados en el coaching (Silins et al., 2002) y empieza a validarse como una herramienta factible de ser utilizada en el ámbito de la educación (Bisquerra, 2008; Gordillo 2008). Entre las diferentes propuestas destacan las siguientes: la primera, se trata de un instrumento de medida de la inteligencia emocional basado en un cuestionario y una serie de ítems que miden aspectos relacionados con las emociones propias del alumnado, la empatía o el autocontrol frente a las críticas, la automotivación o el grado de satisfacción (Rego et al., 2006); y el segundo, un instrumento de evaluación psicológica que puede facilitar información sobre variables importantes relacionadas con el proceso de motivación escolar, atribuciones causales y metas académicas en contextos educativos de enseñanza media y universitaria (Barca et al., 2005).

Concluimos pues, la importancia de la consideración del coaching como 
proceso que puede tener efectos importantes en el desarrollo personal y profesional de las personas a través de la comunicación, favoreciendo una toma de decisiones más adecuada y efectiva, incluso en el afrontamiento de situaciones profesionales que preocupan al alumnado (Álvarez González et al., 2009).

\section{Diseño de la Investigación}

\section{Objetivos, Dimensiones, Subdimensiones e Indicadores}

El objetivo fundamental es la construcción de un sistema de indicadores que permita medir la evolución de las habilidades personales después de un proceso de coaching. Junto a este general destacamos, a continuación, otros que están directamente relacionados con las dimensiones que analizamos en el proyecto.

- Analizar la influencia del coaching universitario en el aprendizaje socioafectivo (dimensión socioafectiva).

- Conocer cómo evolucionan y cambian las variables dinámicas de la personalidad (dimensión sociocognitiva).

- Detectar la influencia del coaching en la planificación y organización de las metas (dimensión ejecutiva).

- Facilitar que el alumnado adquiera habilidades personales y competencias a través del coaching que le ayuden a profundizar en su autoconocimiento (dimensión social).

- Determinar cómo el coaching incide en la gestión personal y académica del alumnado en el seno de la institución universitaria (dimensión comunicativa).

- Comprender las interacciones que se producen entre el alumnado y la institución, analizando la influencia del coaching en dichas sinergias (dimensión identificativa).

Para la propuesta de las dimensiones se ha realizado un intenso trabajo de análisis bibliográfico lo que ha permitido identificar cuáles son las más utilizadas por la comunidad científica cuando se ha abordado el estudio de las habilidades personales. Así se han tenido en cuenta tres ámbitos determinantes que inciden en el rendimiento personal y académico del alumnado, que son: la percepción que tiene el estudiante de sí mismo; la 


\section{Lozano-Pérez \& Trinidad-Requena-Capital Cultural}

percepción de su entorno cercano; y la percepción que tiene el alumnado sobre la influencia de la institución en su crecimiento personal y profesional.

Finalmente, las dimensiones objeto de análisis han sido las siguientes: dimensión socioafectiva, dimensión sociocognitiva, dimensión ejecutiva, dimensión social, dimensión comunicativa y dimensión identificativa. Para facilitar el tránsito del constructo teórico (con su correspondiente definición operativa) al empírico, estas dimensiones se han subdividido a su vez en subdimensiones (por ejemplo, la dimensión comunicativa se subdivide en asertividad grupal, inteligencia social y participación).

Para medir empíricamente las subdimensiones se construyeron una serie de indicadores que básicamente consisten en una serie de ítems ante los cuales se muestra el grado de acuerdo o desacuerdo en una escala tipo Likert (Morales, 1998) con cinco opciones de respuesta (por ejemplo, "el sentido de pertenencia a la universidad" se mide con los indicadores o variables empíricas "Soy consciente de pertenecer a una universidad muy reconocida en el ámbito internacional" y "Cuando escucho noticias buenas en los medios de comunicación de la Universidad de Granada me siento orgulloso/a").

La participación se mide con los indicadores o variables empíricas "Participo en las asociaciones estudiantiles porque considero que es importante estar representado/a" y "Soy de esas personas que van por libre en mi Facultad"). La tabla 1 muestra la totalidad del sistema de indicadores. 


\section{Tabla 1}

Índices de homogeneidad y fiabilidad del cuestionario

\begin{tabular}{|c|c|c|c|c|}
\hline $\begin{array}{c}\text { Dimensiones o } \\
\text { variables teóricas }\end{array}$ & $\begin{array}{l}\text { Subdimensión } \\
\text { teórica }\end{array}$ & Indicador o variable empírica & $\begin{array}{c}\text { Índice } \\
\text { de } \\
\text { Homog } \\
\text { eneidad }\end{array}$ & $\begin{array}{c}\text { Alfa de } \\
\text { Cronbach } \\
\text { al eliminar } \\
\text { el ítem } \\
\end{array}$ \\
\hline \multirow[t]{7}{*}{$\begin{array}{c}\text { DIMENSIÓN } \\
\text { SOCIOAFECTIVA }\end{array}$} & ACTITUD & $\begin{array}{l}\text { P.1.1.1 Me ocupo por mostrar mi mejor } \\
\text { cara cada día. }\end{array}$ & 0.372 & 0.841 \\
\hline & & $\begin{array}{l}\text { P.1.1.3 Intento participar en clase } \\
\text { aportando mis propuestas e ideas. }\end{array}$ & 0.252 & 0.846 \\
\hline & $\begin{array}{l}\text { INTELIGENCIA } \\
\text { EMOCIONAL }\end{array}$ & $\begin{array}{l}\text { P.1.3.3 Cuando te relacionas con los } \\
\text { demás percibes que tienes habilidades } \\
\text { para entender sus emociones. }\end{array}$ & 0.239 & 0.845 \\
\hline & CREATIVIDAD & $\begin{array}{l}\text { P.1.6.2 Me entusiasma buscar nuevas } \\
\text { alternativas a cómo hacer las cosas. }\end{array}$ & 0.374 & 0.840 \\
\hline & $\begin{array}{l}\text { APERTURA A LA } \\
\text { EXPERIENCIA }\end{array}$ & $\begin{array}{l}\text { P.1.7.1 Soy una persona preocupada por } \\
\text { aprender todo lo que pueda dentro de la } \\
\text { Universidad. }\end{array}$ & 0.349 & 0.840 \\
\hline & & $\begin{array}{l}\text { P.1.7.2 Creo que el futuro será mejor que } \\
\text { el presente. }\end{array}$ & 0.225 & 0.844 \\
\hline & AUTOEFICACIA & $\begin{array}{l}\text { P.1.8.B.1 Considero que puedo superar } \\
\text { con éxito cualquier examen que realice. }\end{array}$ & 0.568 & 0.843 \\
\hline $\begin{array}{c}\text { DIMENSIÓN } \\
\text { SOCIOCOGNITIVA }\end{array}$ & & $\begin{array}{l}\text { P.1.8.B.2 Soy una persona que confía en } \\
\text { sus posibilidades en los momentos } \\
\text { importantes. }\end{array}$ & 0.633 & 0.840 \\
\hline DIMENSIÓN & MOTIVACIÓN & $\begin{array}{l}\text { P.2.1.A.1 Normalmente me motivo a mí } \\
\text { mismo/a para dar lo mejor. }\end{array}$ & 0.445 & 0.840 \\
\hline \multirow[t]{5}{*}{ EJECUTIVA } & $\begin{array}{l}\text { INTRINSECA } \\
\text { (MOTIVACIÓN) }\end{array}$ & $\begin{array}{l}\text { P.2.1.A.2 Sé que me merezco que las } \\
\text { cosas me vayan bien. }\end{array}$ & 0.285 & 0.843 \\
\hline & $\begin{array}{l}\text { TOMA DE } \\
\text { DECISIONES }\end{array}$ & $\begin{array}{l}\text { P.3.1.2 En momentos decisivos } \\
\text { importantes, pido consejo a amigos/as o } \\
\text { familiares. }\end{array}$ & 0.243 & 0.845 \\
\hline & $\begin{array}{l}\text { RESOLUCIÓN DE } \\
\text { PROBLEMAS }\end{array}$ & $\begin{array}{l}\text { P.3.2.1 Cuando tengo un problema me } \\
\text { enfrento a él inmediatamente. }\end{array}$ & 0.448 & 0.838 \\
\hline & $\begin{array}{l}\text { CAPACIDAD DE } \\
\text { ANTICIPACIÓN }\end{array}$ & $\begin{array}{l}\text { P.3.4.1 Suelo llevar al día mis clases } \\
\text { porque es una manera de conseguir } \\
\text { buenos resultados. }\end{array}$ & 0.267 & 0.844 \\
\hline & $\begin{array}{l}\text { GESTIÓN DEL } \\
\text { ESTRÉS }\end{array}$ & $\begin{array}{l}\text { P.3.6.1 Sé reconocer bien situaciones de } \\
\text { estrés que pueden afectar a mi } \\
\text { rendimiento. }\end{array}$ & 0.373 & 0.843 \\
\hline \multirow[t]{4}{*}{$\begin{array}{c}\text { DIMENSIÓN } \\
\text { SOCIAL } \\
\text { (LA INSTITUCIÓN) }\end{array}$} & $\begin{array}{l}\text { OPTIMISMO Y } \\
\text { BIENESTAR } \\
\text { SUBJETIVO }\end{array}$ & $\begin{array}{l}\text { P.3.7.2 Pienso que todas las dificultades } \\
\text { se pueden solucionar. }\end{array}$ & 0.483 & 0.841 \\
\hline & RESILIENCIA & $\begin{array}{l}\text { P.3.8.2 Por más difíciles que sean las } \\
\text { situaciones, soy capaz de enfrentarme a } \\
\text { ellas. }\end{array}$ & 0.549 & 0.841 \\
\hline & $\begin{array}{l}\text { COHESIÓN } \\
\text { GRUPAL }\end{array}$ & $\begin{array}{l}\text { P.4.1.1 Cuando hay una discusión entre } \\
\text { mis compañeros/as procuro mediar para } \\
\text { llegar a un acuerdo. }\end{array}$ & 0.385 & 0.842 \\
\hline & $\begin{array}{l}\text { CONDUCTA } \\
\text { PROSOCIAL }\end{array}$ & $\begin{array}{l}\text { P.4.2.1 Normalmente participo con mi } \\
\text { ayuda en actividades que organizan otras } \\
\text { personas. }\end{array}$ & 0.400 & $\begin{array}{c}0.844 \\
\text { (continued) }\end{array}$ \\
\hline
\end{tabular}




\section{Lozano-Pérez \& Trinidad-Requena-Capital Cultural}

\section{Tabla 1}

Índices de homogeneidad y fiabilidad del cuestionario (continued)

\begin{tabular}{|c|c|c|c|c|}
\hline $\begin{array}{c}\text { Dimensiones o } \\
\text { variables teóricas }\end{array}$ & $\begin{array}{l}\text { Subdimensión } \\
\text { teórica }\end{array}$ & Indicador o variable empírica & $\begin{array}{c}\text { Índice } \\
\text { de } \\
\text { Homog } \\
\text { eneidad }\end{array}$ & $\begin{array}{c}\text { Alfa de } \\
\text { Cronbach } \\
\text { al eliminar } \\
\text { el ítem } \\
\end{array}$ \\
\hline $\begin{array}{l}\text { DIMENSIÓN } \\
\text { SOCIAL }\end{array}$ & \multirow[t]{2}{*}{ COMPETENCIAS } & $\begin{array}{l}\text { P.4.3.1 Los demás me consideran una } \\
\text { persona en la que se puede confiar. }\end{array}$ & 0.356 & 0.843 \\
\hline (LA INSTITUCIÓN) & & $\begin{array}{l}\text { P.4.3.3 Cuando hay que tomar } \\
\text { decisiones en grupo me preocupo de que } \\
\text { todo el mundo participe con su opinión. }\end{array}$ & 0.316 & 0.843 \\
\hline \multirow{5}{*}{$\begin{array}{c}\text { DIMENSIÓN } \\
\text { COMUNICATIVA } \\
\text { (LA INSTITUCIÓN) }\end{array}$} & $\begin{array}{l}\text { ASERTIVIDAD } \\
\text { GRUPAL }\end{array}$ & $\begin{array}{l}\text { P.5.1.1 A veces para poder avanzar hay } \\
\text { que decir las cosas claras a la gente, } \\
\text { aunque pueda causar alguna molestia a } \\
\text { alguien. }\end{array}$ & 0.310 & 0.844 \\
\hline & CREATIVIDAD & $\begin{array}{l}\text { P.5.1.2 Soy capaz de aclarar con los } \\
\text { demás algún problema sin causar tensión } \\
\text { ni enfados. }\end{array}$ & 0.367 & 0.842 \\
\hline & \multirow{3}{*}{$\begin{array}{l}\text { SENTIDO DE } \\
\text { PERTENENCIA A } \\
\text { LA UNIVERSIDAD }\end{array}$} & P.6.1.1 Me gusta hacer gala por donde & 0.439 & 0.842 \\
\hline & & $\begin{array}{l}\text { voy de ser alumno/a de la Universidad } \\
\text { de Granada. }\end{array}$ & & \\
\hline & & $\begin{array}{l}\text { P. } 6.1 .2 \text { Como estudiante, reconozco que } \\
\text { es importante pertenecer a esta } \\
\text { Universidad. }\end{array}$ & 0.597 & 0.839 \\
\hline \multirow{9}{*}{$\begin{array}{c}\text { DIMENSIÓN } \\
\text { IDENTIFICATIVA } \\
\text { (LA INSTITUCIÓN) }\end{array}$} & AUTOEFICACIA & P.6.1.3 Me siento dentro del grupo de & 0.380 & 0.838 \\
\hline & & & & \\
\hline & $\begin{array}{l}\text { (UNIVERSIDAD/ } \\
\text { SOCIEDAD) }\end{array}$ & $\begin{array}{l}\text { P.6.3.2 Formarme en la Universidad me } \\
\text { prepara para ofrecer a la sociedad y a mi } \\
\text { entorno lo mejor de mí. }\end{array}$ & 0.397 & 0.841 \\
\hline & \multirow[t]{2}{*}{$\begin{array}{l}\text { RECONOCIMIENT } \\
\text { O INSTITUCIONAL }\end{array}$} & $\begin{array}{l}\text { P.6.4.1 Soy consciente de pertenecer a } \\
\text { una Universidad muy reconocida en el } \\
\text { ámbito internacional. }\end{array}$ & 0.533 & 0.840 \\
\hline & & $\begin{array}{l}\text { P.6.4.2 Cuando escucho noticias buenas } \\
\text { en los medios de comunicación sobre la } \\
\text { Universidad de Granada me siento } \\
\text { orgulloso/a. }\end{array}$ & 0.576 & 0.839 \\
\hline & $\begin{array}{l}\text { TOMA DE } \\
\text { DECISIONES }\end{array}$ & $\begin{array}{l}\text { P.6.4.3 En nuestra ciudad, considero que } \\
\text { la Universidad es una de las instituciones } \\
\text { más respetadas. }\end{array}$ & 0.530 & 0.840 \\
\hline & $\begin{array}{l}\text { COHERENCIA } \\
\text { CONDUCTUAL }\end{array}$ & $\begin{array}{l}\text { P.6.5.1 La Universidad realmente me } \\
\text { está haciendo mejor persona. }\end{array}$ & 0.594 & 0.841 \\
\hline & $\begin{array}{l}\text { CAPACIDAD DE } \\
\text { ANTICIPACIÓN }\end{array}$ & $\begin{array}{l}\text { P.6.5.2 Me identifico con los valores de } \\
\text { la Universidad. }\end{array}$ & 0.624 & 0.839 \\
\hline & $\begin{array}{l}\text { GESTIÓN DEL } \\
\text { ESTRÉS }\end{array}$ & $\begin{array}{l}\text { P.6.5.3 Sé que en el futuro voy a estar } \\
\text { muy agradecido/a de haber vivido la } \\
\text { experiencia de haber pasado por la } \\
\text { Universidad. }\end{array}$ & 0.343 & 0.841 \\
\hline
\end{tabular}

El sistema de indicadores queda compuesto, por tanto, por seis dimensiones, cuarenta subdimensiones y setenta y ocho indicadores que miden factores de la personalidad del alumnado, por un lado, y factores 
grupales relacionados con la institución, por otro. En el mismo se incluye siete ítems validados para medir sensibilidad emocional, empatía, comprensión de las emociones propias, motivación intrínseca, comprensión de las emociones de los demás y autocontrol (Rego et al., 2005). La enumeración, descripción y definición de los mismos se realiza a continuación.

\section{A. Factores de la personalidad del estudiantado:}

A.1. Dimensión socioafectiva: el aprendizaje socioafectivo es el conjunto de actitudes, decisiones y comportamientos que las personas adoptamos con el fin de reforzar favorablemente nuestras conductas sociales y personales durante el proceso de aprendizaje. Las subdimensiones que la conforman son las siguientes:

- Actitud: es la capacidad que tenemos las personas de enfrentarnos a las circunstancias de nuestra vida cotidiana.

- Asertividad: es la habilidad para decir lo que pensamos y sentimos (creencias, derechos u opiniones) sin perjudicar a los demás ni ser perjudicado.

- Inteligencia emocional: la inteligencia emocional es la capacidad para gestionar (conciliar, percibir, utilizar, comprender y manejar) nuestras emociones y las de los demás.

- Habilidades prosociales (Stassen et al., 1997): son las acciones a través de las cuales se comparten y se ofrecen cuidados que benefician a los demás, sin que el benefactor espere ninguna gratificación personal.

- Gestión de la ansiedad: es una respuesta automática que se produce en nuestro cerebro ante el reconocimiento de un peligro inminente. Está asociada muy frecuentemente a preocupaciones excesivas y suele desencadenar un estado constante de preocupación.

- Creatividad: es una habilidad típica de la cognición humana, que consiste en la capacidad de generar nuevas ideas o conceptos, o de nuevas asociaciones entre ideas y conceptos conocidos, que habitualmente producen soluciones originales.

- Apertura a la experiencia: se refiere a lo abierta que está una persona a vivir una determinada experiencia.

- Expectativas (variables previas a la conducta): son un conjunto de creencias y suposiciones centradas en el futuro y que se consideran 


\section{Lozano-Pérez \& Trinidad-Requena-Capital Cultural}

lo más probable que suceda.

- Percepción de control/Locus de control (Rotter, 1966; Visdómine y Luciano, 2005): es la creencia que tiene una persona sobre la influencia o no de sus acciones en la obtención de un resultado.

- Autoeficacia/Autoeficacia percibida (Bandura, 1997): es el juicio que hace una persona sobre su capacidad para afrontar situaciones concretas.

- Pérdida de control: son situaciones en las que la persona es incapaz de mantener el control sobre su entorno si no alcanza las metas que se propone o bien es sometida a circunstancias o resultados que no ha elegido voluntariamente.

- Incremento de la acción/Reactancia psicológica (Brehm, 1966): conducta que lleva a la persona a experimentar una activación por encima de su nivel cotidiano con la intención de restaurar una posible amenaza de su libertad.

- Decremento de la acción/Indefensión aprendida (Overnier et al., 1967): es un tipo de conducta que se manifiesta cuando las personas se comportan de manera pasiva con la sensación subjetiva de no poder hacer nada para cambiar lo negativo o buscar continuamente el estímulo positivo.

A.2. Dimensión sociocognitiva: incluye las variables de la personalidad dinámicas, están en la base de nuestra personalidad y van variando con la experiencia y por tanto con el tiempo. Esta dimensión está compuesta por las siguientes subdimensiones:

- Motivación: es la atracción hacia un objetivo que provoca que la persona se ponga en acción, compuesta de necesidades, deseos, tensiones, incomodidades y expectativas. Puede ser intrínseca y extrínseca.

- Motivación intrínseca: se expresa cuando se realiza una actividad porque en sí misma es interesante y satisfactoria para la persona (Ryan et al., 2000).

- Motivación extrínseca: implica la realización de actividades para conseguir algo, bien para obtener un premio o para evitar un castigo (Ryan et al., 2000).

- Estilos cognitivos: son una estrategia de funcionamiento mental que permite diferenciar a los sujetos por el modo prevalente de percibir 
el medio, procesar la información, pensar o resolver problemas, aprender y actuar (De la Torre et al., 1991).

- Independencia/dependencia de campo: mide las diferencias individuales en cuanto a la tendencia de percibir las situaciones de manera global o percibirlas de una manera analítica.

- Impulsividad/reflexibidad: mide la característica de los individuos a responder con mayor rapidez o con un grado mayor de reflexión.

- Tolerancia/intolerancia hacia situaciones inusuales: mide las diferencias individuales a aceptar con mayor o menor agrado situaciones inusuales y a tolerar percepciones o experiencias inusuales según lo que sabe o conoce.

A.3. Dimensión ejecutiva: se trata de habilidades cognitivas encubiertas y autodirigidas internamente al servicio de una meta y que son necesarias para planificar, organizar, guiar, revisar, regularizar y evaluar el comportamiento necesario para conseguir dicha meta. La composición de la misma está conformada por las siguientes subdimensiones:

- Toma de decisiones: es un proceso por el cual se elige entre una o más opciones para resolver una situación.

- Resolución de problemas: es un proceso cognitivo de alto nivel que requiere un control de habilidades más básicas con el objetivo de resolver y concluir un problema.

- Atención (nuevos tiempos de atención): mecanismo cognitivo complejo y voluntario sobre la actividad mental y conductual en la que nos centramos en una parte de la información que nos llega a través de los órganos sensoriales.

- Capacidad de anticipación: es la capacidad de las personas para prever las situaciones futuras y actuar en consecuencia para potenciar resultados positivos y atenuar o anular consecuencias desfavorables.

- Concentración: es muy importante en el aprendizaje y se define como un proceso psicológico en el que interviene el razonamiento y consiste en centrar la atención en una actividad, objetivo u objeto discriminando cualquier otro estímulo exterior que pueda interferir en dicha atención.

- Gestión del estrés (valoración cognitiva e intervención): es un estado en el que se producen una serie de respuestas físicas ante 
determinados estímulos repetidos.

- Optimismo y bienestar subjetivo: es una actitud que permite estar en disposición de percibir siempre lo más positivo de todo. El bienestar subjetivo es un concepto extenso que incluye la felicidad, la satisfacción con la vida y el afecto positivo.

- Resiliencia: es la capacidad que tiene una persona para sobreponerse a situaciones críticas y adversas que influyen en su estado emocional.

- Gestión del tiempo: es la estrategia para evaluar, gestionar y aplicar recursos con el objetivo de hacer un buen aprovechamiento del tiempo.

- Responsabilidad: es la actitud y el valor de tomar decisiones conscientes y razonadas con respecto a un compromiso u objetivo, así como de asumir las consecuencias que tengan dichas decisiones.

- Rendimiento personal (subjetividad personal y rendimiento académico): es la evaluación personal propia que se hace para medir el balance entre los medios empleados para conseguir algo y los resultados obtenidos.

B. Factores organizacionales y grupales relacionados con la institución:

B.1. Dimensión social: mide aspectos de interrelación social entre el alumnado y su entorno de iguales, incluida la universidad como ente social. Esta dimensión está compuesta por las siguientes subdimensiones:

- Cohesión grupal: es un proceso clave en la formación y desarrollo de los equipos de personas. Carron et al., (1998) la definen como un proceso dinámico que se refleja en la tendencia del grupo a mantenerse y permanecer unido en la búsqueda de sus objetivos instrumentales y/o para la satisfacción de las necesidades afectivas de los miembros.

- Conducta prosocial: es un comportamiento que va destinado a beneficiar a otros y que tiene consecuencias sociales positivas. Es necesario que este comportamiento contenga una intención de beneficiar a otras personas y que haya una libertad de elección.

- Competencias: se entiende como la capacidad o habilidad de la persona para relacionarse con el entorno con éxito logrando sus objetivos.

B.2. Dimensión comunicativa: mide los mecanismos de comunicación 
que se establecen en la interrelación entre el alumnado y su entorno de iguales, incluida la universidad como ámbito que influye y potencia la libertad de expresión y el vínculo comunicativo. Las subdimensiones que considera son las tres siguientes:

- Asertividad grupal: es la habilidad para ser asertivos en el marco del grupo.

- Inteligencia social: propuesta por Gardner (1983) en sus inteligencias múltiples, son un conjunto de habilidades que nos permiten relacionarnos con efectividad con el resto de personas.

- Participación: la podemos definir como el grado de implicación e interacción de una persona en el conjunto de actividades, acciones o iniciativas de las que forma parte.

B.3. Dimensión identificativa: comprende el conjunto de rasgos y características que posee una persona, entidad o comunidad que la distingue de otra y está integrada por las siguientes subdimensiones:

- Sentido de pertenencia a la universidad: es el sentimiento de arraigo emocional que experimenta una persona hacia la universidad a través del grado de identidad, de la emoción predominante, del compromiso establecido, etc.

- Grado de satisfacción: es una medida personal de la valoración que hace el alumnado con respecto a la relación establecida con la universidad, en términos de lealtad, de agradecimiento, de conformidad, etc.

- Conciencia de contribución (universidad/sociedad): se puede definir como la estimación subjetiva y práctica que hace la persona de su aportación al entorno cercano, a la universidad y a la sociedad en general derivado de su estancia de formación universitaria.

- Reconocimiento institucional: se puede considerar como una aproximación a la labor de la universidad como entidad e institución en su compromiso académico, desde la perspectiva del alumnado.

- Coherencia conductual: se refiere al equilibrio y simetría que muestra una persona formada en la universidad con respecto a los propios valores intrínsecos de la institución. 


\section{Lozano-Pérez \& Trinidad-Requena-Capital Cultural}

\section{Diseño de la Investigación: el Experimento}

La metodología aplicada en la investigación ha sido experimental, denominada antes/después, en la que el grupo experimental de coaching es observado antes de la introducción del estímulo y después de la introducción, y así poder comparar los resultados (Grawitz, 1975). En el proyecto de innovación docente todo el alumnado seleccionado ha participado en el proceso de coaching, con la salvedad que unos lo inician en el mes de noviembre-diciembre de 2016 y otros en febrero-abril de 2017, y el cuestionario final se realiza en el mes de mayo 2017. Se establece un grupo de control de 53 estudiantes que tienen las mismas características que el grupo experimental pero que como es lógico no han recibido el proceso de coaching.

La aplicación del proceso de coaching ha sido realizada por coaches de la Escuela de Formación Integral en Coaching (EFIC) y de la Internacional Coaching Federation (ICF) a través de una programación de sesiones presenciales (un mínimo de tres) con una duración de hora y media cada una.

Se ha asumido que los cambios que puedan producirse en el interés de las personas, en sus opiniones o en su situación personal no varían sustancialmente en tan corto periodo (tres meses) de forma no intencionada o circunstancial ("ceterisparibus" para las demás variables), y con ello poder medir los cambios que se produce en sus habilidades personales derivadas de la realización del proceso de coaching.

\section{Técnicas de Producción de Datos y Trabajo de Campo}

La técnica de producción de datos cuantitativa utilizada ha sido la encuesta. Se ha construido un cuestionario con 78 preguntas para medir las habilidades personales y cuatro preguntas sociodemográficas, en concreto sexo, edad, estudios de los padres y facultad de pertenencia. Además, en la segunda aplicación del cuestionario (grupo después), se agregaron al mismo seis preguntas abiertas para detectar cualitativamente los aspectos positivos y los aspectos a mejorar en el proceso de coaching.

Para verificar el control y la calidad de nuestra herramienta metodológica, se ha realizado un pretest a un número reducido de 40 
estudiantes de la Universidad de Granada, detectándose en el cuestionario inicial algunos errores relativos fundamentalmente a la falta de comprensión de algunos de los ítems propuestos. En el pretest, también se observa que la duración media de este tipo de cuestionario debe situarse en torno a los quince-veinte minutos, ya que se aprecia una disminución de la atención después de este tiempo, relacionado con la utilización sistemática y un tanto repetitiva de las escalas tipo Likert.

El cuestionario definitivo ha sido autoadministrado, en las dependencias de la Facultad de Derecho de la Universidad de Granada, por el propio alumnado que participan en el proceso de coaching, previa explicación por parte de los investigadores de la forma en la que debe cumplimentarse. En todo momento los investigadores han resuelto "in situ" cualquier duda presentada por los encuestados y una vez realizado el cuestionario ha sido revisado delante del encuestado por parte del investigador, por si alguna cuestión no había sido debidamente cumplimentada. Con todos los componentes del equipo de investigación presentes en la aplicación de los cuestionarios autoadministrados se estableció un protocolo de acción común (briefing) para solventar las posibles dudas o consultas del estudiantado durante la realización del cuestionario, con el fin de no introducir sesgos en las respuestas.

La investigación tiene una finalidad exploratoria con el objeto de crear un instrumento de medida adecuado. Se ha encuestado a un número suficiente de estudiantes 203, pero no representativo estadísticamente de la población universitaria de la Universidad de Granada.

\section{Fiabilidad y Validez del Instrumento de Medición}

El control del instrumento que se pretende utilizar en la investigación debe ajustarse a los conceptos, para que realmente mida aquello que pretendemos medir, es decir, es imprescindible comprobar si este instrumento tiene la validez y fiabilidad necesaria (Ruiz Olabuénaga, 1999).

La validez hace referencia a la relación que ha de existir entre el concepto teórico y el indicador o indicadores empíricos, por tanto, el investigador ha de comprobar si los indicadores realmente "indican" lo que se pretende que indiquen, si miden correctamente el significado dado al concepto teórico en consideración. Por otro lado, la fiabilidad es la capacidad de obtener 


\section{Lozano-Pérez \& Trinidad-Requena-Capital Cultural}

resultados consistentes en mediciones sucesivas del mismo fenómeno, es decir, que los resultados logrados en mediciones repetidas (del mismo concepto) han de ser iguales para que la medición sea fiable (Cea D' Ancona, 1999).

Fiabilidad y validez son aspectos muy importantes para juzgar y comprobar la bondad de las escalas y medidas. Ambas están muy relacionadas, de manera que se repercuten mutuamente, por ello ha sido uno de los aspectos en los que más se ha incidido en el proceso de investigación (Latiesa, 2001).

Además de tener presentes criterios estándares a la hora de realizar la investigación como son la rigurosidad en la elaboración del cuestionario, la formación de los aplicadores o el seguimiento y control exhaustivo del trabajo de campo, hemos realizado las pruebas de validez de constructo con el fin de corroborar la estructura dimensional del cuestionario y que sirva para la posterior interpretación de los resultados. Para ello, con anterioridad se ha calculado la media de adecuación muestral KMO. En la investigación este estadístico arrojó un valor de 0,6, valor superior a 0,5 lo que indica que las correlaciones entre dichas variables son significativas y que por lo tanto tendría sentido realizar un análisis factorial de las relaciones entre las variables con los datos de la muestra. El resultado del estadístico anterior se confirma con la prueba de esfericidad de Barlett, en nuestro caso la significación de la prueba ofrece un valor inferior a 0,05 , y ratifica la necesidad de realizar el análisis de la estructura de las dimensiones del cuestionario o lo que es igual, el análisis factorial del mismo.

Para determinar la fiabilidad del cuestionario se han realizado los análisis de consistencia interna y el análisis de la capacidad de discriminación de los ítems. En relación a la consistencia interna se suele calcular el coeficiente alfa de Cronbach que asume que los ítems miden un mismo constructo y están muy correlacionados. El valor del coeficiente para el conjunto de ítems ha sido de 0,76, que es considerado como aceptable. Para el análisis de la capacidad de discriminación de los ítems del cuestionario hemos utilizado el índice de homogeneidad que nos informa del grado en que un ítem está midiendo lo mismo que la prueba globalmente y por lo tanto cómo contribuye a la consistencia interna del cuestionario. Los ítems con índices bajos de homogeneidad inferiores a 0,2 miden algo diferente a lo que pretende el cuestionario en su conjunto, por lo que podemos eliminarlos del 
cuestionario y resultan ser ítems indiferenciadores ya que aportan escasa información sobre lo que estamos midiendo, por lo que no tiene sentido combinarlos con los demás (McIver et al., 1981).

En nuestro caso, el índice de homogeneidad corregido elimina más de la mitad de los ítems originales quedando el cuestionario con 32 ítems que resultan ser diferenciadores. Así la estructura dimensional del cuestionario ha quedado tal y como refleja la tabla 1 . Como se puede observar, algunas de las subdimensiones establecidas inicialmente no cuentan con indicadores para su posible medición, y pasamos de 40 subdimensiones originales a 19. Aunque se ha conseguido una buena consistencia interna del cuestionario con la aplicación del índice de homogeneidad, la estructura dimensional del mismo ha quedado comprometida y nos confirma que se hace preciso realizar un análisis factorial para replantearnos la conceptualización de las dimensiones y subdimensiones.

La tabla 2 muestra el resultado del análisis factorial exploratorio que se ha realizado a la nueva estructura dimensional resultante de la eliminación de más de la mitad de los ítems originales. Como buscamos el número de factores que mejor se adapte al modelo propuesto, la extracción de los mismos no se ha hecho para un número predeterminado de factores sino para aquellos con autovalores mayores que 1, en nuestro caso fueron 11 factores que explican el $66,040 \%$ de la varianza total. La estructura dimensional del modelo ha quedado tal y como muestra la tabla 3 . 


\section{Lozano-Pérez \& Trinidad-Requena-Capital Cultural}

Tabla 2

Varianza total explicada por el nuevo modelo

\begin{tabular}{lccc}
\hline Factor & Autovalor & \% de la varianza & \% acumulado \\
\hline 1 & 5,800 & 18,124 & 18,124 \\
2 & 3,261 & 10,284 & 28,408 \\
3 & 2,094 & 6,544 & 34,952 \\
4 & 1,765 & 5,515 & 40,467 \\
5 & 1,428 & 4,464 & 44,930 \\
6 & 1,301 & 4,066 & 48,997 \\
7 & 1,208 & 3,774 & 52,770 \\
8 & 1,123 & 3,509 & 56,279 \\
9 & 1,095 & 3,422 & 59,701 \\
10 & 1,020 & 3,188 & 62,889 \\
11 & 1,008 & 3,151 & 66,040 \\
\hline
\end{tabular}

El nuevo modelo ha superado al inicial en poder explicativo y en adecuación de los datos al análisis factorial mejorando la media de adecuación muestral KMO $(0,741)$, también en relación a la fiabilidad del nuevo modelo (consistencia interna) la alfa de Crombach total arrojó un valor de 0,846 , valor considerado como muy bueno.

Como resultado del análisis de la matriz de componentes las variables se han agrupado tal y como refleja la tabla 3, además de los 32 ítems, se elimina uno ya que su valor absoluto es menor de 0,40 que suele ser el valor mínimo admitido. El nuevo sistema de indicadores surgido después del análisis de la calidad del sistema inicial posee la información necesaria para identificar posibles cambios en los ítems después de la realización del proceso de coaching, y así sigue resultando factible analizar la eficacia del coaching en diferentes ámbitos de la personalidad de los estudiantes. 


\section{RISE - International Journal of Sociology of Education, 8(1) 95}

\section{Tabla 3}

\section{Sistema de indicadores después del análisis de la calidad del sistema inicial}

NUEVOS FACTORES

FACTOR 1. SENTIDO DE

PERTENENCIA Y RECONOCIMIENTO

INSTITUCIONAL (INSTITUCIÓN)

FACTOR 2. AUTOEFICACIA Y RESOLUCIÓN DE PROBLEMAS (EJECUTIVAS)

FACTOR 3. CONDUCTA PROSOCIAL Y COHESIÓN GRUPAL

FACTOR 4. CONCIENCIA DE CONTRIBUCIÓN A LA UNIVERSIDAD: FORMACIÓN Y VALORES

FACTOR 5. APERTURA A LA EXPEIENCIA Y CAPACIDAD DE ANTICIPACIÓN

FACTOR 6. OPTIMISMO Y GESTIÓN DEL ESTRÉS

FACTOR 7. INTERACCIÓN SOCIAL

FACTOR 8. MOTIVACIÓN INTRINSECA

FACTOR 9. COMPETENCIAS Y

ASERTIVIDAD

FACTOR 10. INTELIGENCIA

EMOCIONAL (EMPATÍA)
P.6.4.1 Soy consciente de pertenecer a una Universidad muy reconocida en el ámbito internacional.

P.6.4.2 Cuando escucho noticias buenas en los medios de comunicación sobre la Universidad de Granada me siento orgulloso/a.

P.6.4.3 En nuestra ciudad, considero que la Universidad es una de las instituciones más respetadas.

P.6.1.1 Me gusta hacer gala por donde voy de ser alumno/a de la Universidad de Granada.

P.6.1.2 Como estudiante, reconozco que es importante pertenecer a esta Universidad. P.6.3.2 Formarme en la Universidad me prepara para ofrecer a la sociedad y a mi entorno lo mejor de mí.

P.1.8.B.1 Considero que puedo superar con éxito cualquier examen que realice. P.1.8.B.2 Soy una persona que confía en sus posibilidades en los momentos importantes.

P.3.2.1 Cuando tengo un problema me enfrento a él inmediatamente.

P.3.7.2 Pienso que todas las dificultades se pueden solucionar.

P.3.8.2 Por más difíciles que sean las situaciones, soy capaz de enfrentarme a ellas.

P.1.1.1 Me ocupo por mostrar mi mejor cara cada día.

P.4.1.1 Cuando hay una discusión entre mis compañeros/as procuro mediar para llegar a un acuerdo.

P.4.2.1 Normalmente participo con mi ayuda en actividades que organizan otras personas.

P.5.1.2 Soy capaz de aclarar con los demás algún problema sin causar tensión ni enfados.

P.6.5.1 La Universidad realmente me está haciendo mejor persona.

P.6.5.2 Me identifico con los valores de la Universidad.

P.6.5.3 Sé que en el futuro voy a estar muy agradecido/a de haber vivido la experiencia de haber pasado por la Universidad.

P.1.7.1 Soy una persona preocupada por aprender todo lo que pueda dentro de la Universidad.

P.3.4.1 Suelo llevar al día mis clases porque es una manera de conseguir buenos resultados.

P.1.1.3 Intento participar en clase aportando mis propuestas e ideas.

P.1.7.2 Creo que el futuro será mejor que el presente.

P.3.6.1 Sé reconocer bien situaciones de estrés que pueden afectar a mi rendimiento. P.1.6.2 Me entusiasma buscar nuevas alternativas a cómo hacer las cosas.

P.4.3.3 Cuando hay que tomar decisiones en grupo me preocupo de que todo el mundo participe con su opinión.

P.5.1.1 A veces para poder avanzar hay que decir las cosas claras a la gente, aunque pueda causar alguna molestia a alguien.

P.2.1.A.1 Normalmente me motivo a mí mismo/a para dar lo mejor.

P.2.1.A.2 Sé que me merezco que las cosas me vayan bien.

P.4.3.1 Los demás me consideran una persona en la que se puede confiar.

P.1.3.3 Cuando te relacionas con los demás percibes que tienes habilidades para entender sus emociones.

P.3.1.2 En momentos decisivos importantes, pido consejo a amigos/as o familiares. 


\section{Lozano-Pérez \& Trinidad-Requena-Capital Cultural}

\section{Discusión}

En esta investigación la principal técnica de análisis multivariante utilizada ha sido el análisis factorial exploratorio. Su aplicación permite identificar las interrelaciones entre las variables y reducirlas a un número menor que retenga la mayor parte de la varianza de las variables originales (Hair et al., 2007). Pero los métodos para analizar la estructura dimensional de un sistema de indicadores son variados y aunque el análisis factorial exploratorio es la técnica más utilizada existen una serie de limitaciones vinculadas a aspectos tales como el tamaño de la muestra, el número de variables utilizadas por factor, el carácter continuo o discreto de las mismas o el coeficiente de correlación empleado (Garrido et al., 2013).

Por ello hemos confrontando los resultados obtenidos mediante el análisis factorial con otro método como es el "parallel analysis" (Horn, 1965) en torno al cual existe un gran conceso en su implementación, ya que es considerado una solución óptima para la identificación de factores. El programa utilizado ha sido el Montecarlo PCA for Parallel Analysis (Watkins, 2000), que es un software que genera múltiples muestras del mismo tamaño, y calcula la media de los autovalores iniciales (eigenvalues) para dichas muestras. Comparando los eigenvalues resultantes del programa Montecarlo PCA con los obtenidos con el SPSS, podemos seleccionar el número de factores más adecuado a retener, y si el autovalor del factor de SPSS es mayor que el obtenido con el programa Montecarlo PCA se acepta dicho factor (Zwick y Velicer, 1986; Lautenschalager, 1989; Velicer et al., 2000).

Los resultados obtenidos para el caso de estudio muestran las mismas conclusiones que con el procedimiento clásico aplicado en la investigación, ya que también retiene 11 factores tal y como muestra la tabla 4 . 


\section{Tabla 4}

Comparación eigenvalues Montecarlo PCA /SPSS. Aceptación de Factores

\begin{tabular}{llll}
\hline $\begin{array}{c}\text { Número de } \\
\text { Factores }\end{array}$ & Eigenvalue SPSS & \multicolumn{1}{c}{$\begin{array}{c}\text { Eigenvalue } \\
\text { Montecarlo } \\
\text { PCA }\end{array}$} & Decisión \\
\hline 1 & 8,191 & 2,504 & Aceptado \\
2 & 5,315 & 2,382 & Aceptado \\
3 & 4,553 & 2,281 & Aceptado \\
4 & 3,586 & 2,209 & Aceptado \\
5 & 3,474 & 2,136 & Aceptado \\
6 & 2,695 & 2,081 & Aceptado \\
7 & 2,278 & 2,017 & Aceptado \\
8 & 2,002 & 1,960 & Aceptado \\
9 & 1,927 & 1,910 & Aceptado \\
10 & 1,874 & 1,859 & Aceptado \\
11 & 1,801 & 1,801 & Aceptado \\
12 & 1,705 & 1,758 & No aceptado \\
13 & 1,634 & 1,712 & No aceptado \\
\hline
\end{tabular}

\section{Conclusión}

El objetivo principal de este texto ha sido mostrar cómo se ha articulado teórica y metodológicamente un proyecto de innovación docente dirigido a comprobar la eficacia de un proceso de coaching en el ámbito educativo universitario.

A nivel teórico, se ha partido de la consideración del coaching como herramienta de gestión y aplicación al ámbito de la educación superior, que se ha visto modificada por la adaptación al Plan Bolonia. El Espacio Europeo de Educación Superior y el coaching comparten un mismo enfoque de trabajo basado en la autonomía del estudiante, el autoaprendizaje y el 


\section{Lozano-Pérez \& Trinidad-Requena-Capital Cultural}

desarrollo de competencias en el proceso de aprendizaje, de ahí la idoneidad de disponer de un sistema de indicadores válido y fiable que mida las mejoras en estos aspectos.

A nivel metodológico, una de las cuestiones centrales planteadas desde el equipo de investigación ha hecho referencia a la eficacia del proceso de coaching, por ello, ante la limitación generada por la ausencia de cuestionarios validados que nos permitiesen evaluar el proceso de coaching y detectar los cambios que este podría producir, se ha diseñado un cuestionario "ad hoc". Paralelamente otra preocupación surgida viene generada por las restricciones impuestas por el tipo de metodología que se emplea para la realización del análisis factorial tal y como se pone de manifiesto en el apartado de la discusión.

El cuestionario que finalmente se ha diseñado supone un paso para conseguir una herramienta que mida la eficacia del coaching en aspectos socioafectivos, sociocognitivos, ejecutivos, sociales, identificativos con la institución y comunicativos del alumnado universitario, dado que hasta la fecha no hay constancia de un instrumento similar.

\section{Agradecimientos}

La investigación en la que está basado el presente artículo está vinculado a un Proyecto de Innovación Docente, financiado por la Universidad de Granada, titulado "Potenciando las capacidades y habilidades del alumnado a través del coaching académico con especial atención a la integración de alumnos con discapacidad o necesidades educativas especiales" y coordinado por la Dra. María del Mar Méndez Serrano.

\section{Referencias}

Álvarez González, M., \& Obiols Soler, M. (2009). El proceso de toma de decisiones profesionales a través del coaching. Electronic Journal of Research in Educational Psychology, 7(2), 877-900. doi:

10.25115/ejrep.v7i18.1323

Asociación Española de Coaching (ASESCO) (2016). Coaching real para necesidades reales. Recuperado de http://www.asescoaching.org/elcoaching/

Bandura, A. (1997). Self-efficacy: the exercise of control. New York, W.H. Freeman and Company. 
Barca, A., Porto, A., Santorum, R. \& Barca, E. (2005). Motivación académica, orientación a metas y estilos atribucionales: la escala CEAP -48. Revista de Psicología y Educación, 1(2), 103-136.

Bisquerra, R. (2008). Coaching. Un reto para los orientadores. Revista de Orientación Psicopedagógica, 19(2), 163-170.

Bou Pérez, J.F. (2007). Coaching para docentes. Editorial Club Universitari. Brehm, J.W. (1966). A theory of psychological reactance. New York, Academic Press.

Camacho, J. (2002). Estadística con SPSS para Windows. Madrid. Editorial RA-MA.

Carron, A. V., Brawley, L. R., \& Widmeyer, W. N. (1998). The measurement of cohesiveness in sport groups. In J. L. Duda, (Ed.), Advances in sport and exercise psychology measurement (pp.213226). Morgantown, WV, Fitness Information Technology.

Cea D’Ancona, M.A. (1998). Metodología Cuantitativa. Madrid, Editorial Síntesis.

Coutu, D., \& Kauffman, D. (2009). What Can Coaches Do for You? Harvard Business Review, January, 91-98.

De La Torre, S. \& Mallart, J. (1991). Estilos Cognitivos y Currículo. Un Modelo de Mejorar la Instrucción. Revista de Pedagogía, 43(1), 3954.

De Meuse, Kenneth P., Dai, Guangrong \& Lee, Robert J.(2009). Evaluating the effectiveness of executive coaching: beyond ROI? Coaching: An International Journal of Theory, Research and Practice, 2(2), 117134. doi: 10.1080/17521880902882413

Dilts, R. (2004). Coaching. Herramientas para el cambio, Urano. Echeverría, R. (2013). Ética y coaching ontológico. Ediciones Granica.

Gaffner, D. C., \& Hazler, R. J. (2002). Factors Related to Indecisiveness and Career Indecisión in Undecided College Students. Journal of College Student Development, 43 (3), 317-326.

doi: $10.1177 / 1069072712454698$

Gallwey, T. (2006). El juego interior del tenis. Editorial Sirio. Gardner, H. (1983). Frames of Mind: The theory of Multiple intelligences. New York, Basic Books, Harper Collins Publisher Inc. Garrido, L.E, Abad F.J, \& Posonda, V. (2013). A new look at Horn's parallel analysis with ordinal variables. American Psychological 
Association, 18, 4, 454-474. doi: 10.1037/a0030005

Gordillo, M.V. (2008). Nuevas perspectivas en orientación: del counseling al coaching. Revista digital Orientación y sociedad, 8, 333.

Grawitz M. (1975). Métodos y técnicas de las ciencias sociales, Tomo II.

Barcelona, Editorial Hispano Europea.

Hair J. F., Anderson, R. E., Tatham, R. L.; \& Black, W. C. (2007). Análisis multivariante. Madrid. Editorial Prentice Hall.

Horn, J. L. (1965). A rationale and test for the number of factors in factor analysis. Psychometrika, 30, 179-185. doi: 10.1007/BF02289447

International Coaching Federation (ICF) (2016). Global coaching study. Recuperado de http://www.icf-es.com/mwsicf/sobreicf/definicioncoaching-icf-espana

Latiesa, M. (2001). El pluralismo metodológico en la investigación social: ensayos típicos. Granada, Editorial Universidad de Granada.

Lautenschlager, G. J. (1989). A comparison of alternatives to conducting Monte Carlo analyses for determining parallel analys is criteria. Multivariate Behavioral Research, 24, 365-395. doi:

10.1207/s15327906mbr2403_6

Lozano Correa, L. J. (2008). El coaching como estrategia para la formación de competencias profesionales. Escuela de Administración de Negocios, 63, 127-137. doi: 10.21158/01208160.n63.2008.447

Lloret-Segura, S., Ferreres-Traver, A., Hernández-Baeza, A., \& TomásMarco, I. (2014). Exploratory Item Factor Analysis: A practical guide revised and up-dated. Anales De Psicología / Annals of Psychology, 30(3), 1151-1169. doi: 10.6018/analesps.30.3.19936

McIver, J. P. \& Carmines, E. G. (1981). Unidimensional scaling. Beverly Hills, CA, Sage.

Medina, L. G. \& Perichon, A. M. (2008). Coaching educacional. Una nueva visión de la orientación vocacional. Buenos Aires, Bonum.

Morales, P. (1988). Medición de actitudes en psicología y educación. Construcción de escalas y problemas metodológicos. San Sebastián, Ed. Universidad Pontificia de Comillas.

Overmier, J. B., \& Seligman, M. E. (1967). Effects of inescapable shock upon subsequent escape and avoidance responding. Journal of comparative and physiological psychology, 63(1), 28. doi:

10.1037/h0024166 
Parsloe, E. (1992). Coaching, Mentoring, and Assessing: A Practical Guide to Developing Competence. Kogan Page.

Ravier, L. (2005). Arte y Ciencia del Coaching: su historia, filosofía y esencia. Buenos Aires, Dunken.

Rego, A., \& Fernandes, C. (2005). Inteligencia emocional: desarrollo y validación de un instrumento de medida. Revista Interamericana de Psicología/Interamerican Journal of Psychology, 39,(1), 23-38.

Rotter, J. B. (1966). Generalized expectancies for internal versus external control of reinforcement. Psychological Monographs, 80(1), 1-28. doi: $10.1037 / \mathrm{h} 0092976$

Ryan, R., \& Deci, E. (2000). Self - Determination theory and the facilitation of intrinsic motivation, social development, and well-being. American Psychologist, 55(1), 68-78. doi: 10.1037110003-066X.55.1.68

Sánchez Mirón, B., \& Boronat Mundina, J. (2014). Coaching Educativo: Modelo para el desarrollo de competencias intra e interpersonales. Educación XX1, 17(1), 221-242. doi: 10.5944/educxx1.17.1.1072 Sánchez-Teruel, D. (2009). Actualización en inteligencia emocional. Madrid, CEP.

Stassen, K., \& Thompson, R.A. (1997). Psicología del desarrollo: infancia y adolescencia. Editorial Médica Panamericana. S.A.

Silins, H., \& Mulford, B. (2002). Schools as learning organizations: The case for system, teacher and student learning. The Journal of Educational Administration, 40(5), 425-446. doi:10.1108/09578230210440285

Velicer, W. F. (1976). Determining the number of components from the matrix of partialcorrelations. Psychometrika, 41(3), 321-327. doi: 10.1007/BF02293557

Visdómine-Lozano, J.C., \& Luciano, C. (2006). Locus de control y autorregulación conductual: revisiones conceptual y experimental. International Journal of Clinical and Health Psychology, 6(3), 729751.

Watkins, M.W. (2000). Montecarlo PCA for parallel analysis (computer software). Satate College, PA, Ed \& Psych Associates.

Withmore, J. (2011). Coaching. El método para mejorar el rendimiento de las personas. Ediciones Paidós Ibérica.

Wolk, L. (2009). Coaching. El arte de soplar brasas. Gran Aldea Editores. 


\section{Lozano-Pérez \& Trinidad-Requena-Capital Cultural}

Zeus, P., \& Skiffington, S. (2004). Coaching práctico en el trabajo. Mc Graw Hill.

Zwick, W. R., \& Velicer, W.F. (1986). Comparison of five rules for determining the number of components to retain. Psychological Bulletin, 99(3), 432-442. doi: 10.1037/0033-2909.99.3.432

Jose Luis Paniza Prados is professor at the University of Granada

Inmaculada Puertas Cañaveral is professor at the University of Granada

Jose María Molina Morales is professor at the Escuela de Formación Integral en Coaching

Contact Address: jpaniza@ugr.es 